\title{
Insertion of an extra copy of Xq22.2 into 1 p36 results in functional duplication of the PLP1 gene in a girl with classical Pelizaeus- Merzbacher disease
}

\author{
Julien Masliah-Planchon ${ }^{1,2,3}$, Céline Dupont ${ }^{4}$, George Vartzelis ${ }^{5}$, Aurélien Trimouille ${ }^{1,2}$, Eléonore Eymard-Pierre ${ }^{6,7}$, \\ Mathilde Gay-Bellile ${ }^{6,7}$, Florence Renaldo ${ }^{2,8}$, Imen Dorboz ${ }^{2}$, Cécile Pagan ${ }^{1}$, Samuel Quentin ${ }^{9}$, Monique Elmaleh ${ }^{10}$, \\ Christina Kotsogianni ${ }^{5}$, Elissavet Konstantelou ${ }^{5}$, Séverine Drunat ${ }^{1,2}$, Anne-Claude Tabet ${ }^{4}$ \\ and Odile Boespflug-Tanguy ${ }^{2,8^{*}}$
}

\begin{abstract}
Background: Pelizaeus-Merzbacher disease (PMD) is an X-linked dysmyelinating disorder characterized by nystagmus, hypotonia, ataxia, progressive spasticity, and cognitive decline. PMD classically results from a duplication of a genomic segment encompassing the entire $P L P 1$ gene. Since the $P L P 1$ gene is located in $X q 22$, PMD affects mostly boys.

Methods and results: Here we report the case of a girl with typical PMD. Copy number analysis of the PLP1 locus revealed a duplication of the entire gene and FISH analysis showed that the extra copy of the PLP1 gene was actually inserted in chromosome $1 \mathrm{p} 36$. This insertion of an additional copy of PLP1 in an autosome led to a functional duplication irrespective of the X-inactivation pattern. Subsequent overexpression of PLP1 was the cause of the PMD phenotype observed in this girl. Further sequencing of the breakpoint junction revealed a microhomology and thus suggested a replication based mechanism (such as FoSTeS or MMBIR).

Conclusion: This case emphasizes the susceptibility of the PLP1 locus to complex rearrangement likely driven by the Xq22 local genomic architecture. In addition, careful consideration should be given to girls with classical PMD clinical features since they usually experience complex PLP1 genomic alteration with a distinct risk of inheritance.
\end{abstract}

\section{Background}

Pelizaeus-Merzbacher disease (PMD; MIM\#312080) is a rare X-linked hypomyelinating leukodystrophy related to genomic alterations of the proteolipid protein 1 (PLP1) gene [1]. PLP1 encodes both major proteins of the central nervous system (CNS) myelin, PLP and DM20. PMD is characterized by early nystagmus, hypotonia and ataxia with subsequent signs of neurodegeneration characterized by severe spasticity and cognitive impairment. However, a large clinical spectrum exists from severe

\footnotetext{
* Correspondence: odile.boespflug-tanguy@rdb.aphp.fr

${ }^{2}$ Inserm U1141, Université Paris Diderot, Sorbonne Paris Cité, Hôpital Robert Debré, Paris, France

${ }^{8}$ Neurologie et maladie métabolique, Hôpital Robert Debré, AP-HP, Paris, France

Full list of author information is available at the end of the article
}

forms without motor acquisitions and dystonia (PMD0), to mild forms with acquired walking capacities (PMD3), and the mildest form of PLP-pathies, spastic paraplegia type 2 (SPG2, MIM\#312920). An obvious genotypephenotype correlation has been described in PMD. Missense mutations are rare but usually cause the most severe forms, whereas PLP1 loss-of-function alterations (null mutations and large deletions) lead to the mildest PMD/SPG2 forms. Classical PMD is commonly caused by duplication of a genomic segment containing the entire PLP1 gene. PLP1 gene duplication results in PLP overexpression and subsequent accumulation in the cytoplasm of the myelinating oligodendrocytes leading to cellular stress and CNS myelin defect. A gain of three to five copies of the PLP1 gene have been rarely described and are 
associated with a severe phenotype [2, 3]. PMD affects almost exclusively boys and most females who carry and transmit PLP1 genomic alterations to their affected sons are asymptomatic or manifest mild, late-onset spastic paraplegia or cognitive impairment. Few cases of girls presenting a phenotype of PMD in childhood have been described. Foncesca et al. reported the case of a constitutional translocation $\mathrm{t}(\mathrm{X} ; 22)(\mathrm{q} 22 ; \mathrm{q} 13)$ with an additional PLP1 copy at the breakpoint region in a girl with classical PMD [4]. Yiu et al. reported another case of a PMD girl with an unbalanced chromosomal translocation of PLP1 into 1p36 [5]. Additional cases of girls with PMD due to very large $P L P 1$ gene duplications have been reported [6].

Here we report the case of a 2 year old girl with typical PMD who has an insertion of an extra copy of a genomic segment of Xq22 containing the entire PLP1 gene into chromosome $1 \mathrm{p} 36$. This insertion of an additional active copy of PLP1 in an autosome led to a functional duplication. Further delineation of the breakpoint junction sequence revealed a microhomology and thus support a replication based mechanism such as FoSTeS and MMBIR. Our result further emphasizes the large diversity of the genomic rearrangement involving the PLP1 gene probably based on the complexity of the genomic architecture [7].

\section{Methods}

Multiplex ligation-dependent probe amplification (MLPA) MLPA was conducted according to the supplied manufacturer's specifications using the probemix P022-PLP1 (MRC-holland, Amsterdam, Holland). PCR products were then mixed with formamide (HiDi Formamide, Applied Biosystems, Foster City, CA) and fluorescent Genescan 500 LIZ size standard (Applied Biosystems) prior to analysis using an Applied Biosystems ABI 3130xl capillary sequencer (Applied Biosystems). Data collection and export used GeneMapper software (Applied Biosystems). Peak areas of each fragment were compared to those of a control sample to calculate the gene dosage of each amplicon, including those corresponding to each exon of the PLP1 gene.

\section{$\mathrm{X}$ chromosome inactivation pattern}

The analysis of the $\mathrm{X}$ inactivation pattern was based on extend human androgen receptor (HUMARA) assay proposed Bertelsen et al. [8]. Briefly, $200 \mathrm{ng}$ of leukocyte DNA was digested with HpaII (New England Biolabs, Ipswich, MA) at $37{ }^{\circ} \mathrm{C}$ for $12 \mathrm{~h}$. PCR amplification of four loci (AR, PCSK1N, SLITRK4, and ZDHHC15) was performed on undigested DNA and HpaII digested DNA. PCR products were subsequently analyzed on an ABI 3130xl Genetic Analyzer (Applied Biosystems) using GeneMapper software (Applied Biosystems). The X- inactivation ratios were calculated as previously described and skewed $\mathrm{X}$ chromosome inactivation was considered if this ratio decrease below $20 \%$ in the investigated blood sample [8].

\section{PLP1 gene expression analysis in patient fibroblasts}

Total RNA was isolated from fibroblasts of eight controls without copy number variation of the PLP1 gene, of one positive control with a duplication of $P L P 1$, and of our patient using the guanidinium thiocyanatephenol-chloroform method. Ten microgram was used to generate cDNA using Superscript II first-strand cDNA synthesis kit (Invitrogen, Carlsbad, CA, USA) with oligo (dT) primers, according to the manufacturer's protocol. Our patient and control cDNAs were used for PLP1 gene expression analysis performed by real-time PCR according to previously reported conditions [9]. Specifically designed primers were used to determined total PLP1 gene expression after normalization to the betaglucuronidase gene (GUSB) expression using the $\Delta \Delta \mathrm{Ct}$ method.

\section{Microarray}

Creation of a custom array CGH design was performed using the Agilent software eArray (https://earray.chem. agilent.com/earray/), referred to the GRCh37/hg19 assembly to cover the PLP1 genomic region with maximum resolution. The microarray contained a total of 30,032 probes spanning a $20 \mathrm{Mb}$ region surrounding the PLP1 gene (Agilent Technologies, Santa Clara, CA). Those 30,032 probes were distributed between three groups with various probe densities. The first group includes 15,032 probes encompassing $2 \mathrm{Mb}$ (chrX: 102,000,000-104,000,000; corresponding to a $150 \mathrm{bp}$ average probe spacing) around the PLP1 gene and includes most of the PLP1 duplications described so far $[10,11]$. A second, largest interval contained 10,000 probes surrounding $8 \mathrm{Mb}$ (chrX: 98,000,000-102,000,000 and chrX: 104,000,000-106,000,000; 800 bp average probe spacing). The last interval was designed to encompass the largest PLP1 rearrangement and includes 5,000 probes spanning $10 \mathrm{Mb}$ (chrX: 93,000,00098,000,000 and chrX: 106,000,000-113,000,000; 2000 bp average probe spacing) [6].

A HumanCytoSNP-12 (V2.1) pangenomic microarray (Illumina, San Diego, CA USA) was also performed.

\section{Fluorescence in situ hybridization (FISH)}

Fluorescence in situ hybridization (FISH) was performed on lymphocytes metaphase spreads according to standard protocols. FISH analysis was carried out with the probe RP11-832L2 spanning $183 \mathrm{~Kb}$ (chrX:102,902,650103,085,315), which encompasses the entire PLP1 gene, 
together with a subtelomeric probe of the short arm of chromosome 1 (CEB108/T7).

\section{Genome walker}

The Universal Genome Walking Kit (Clontech, Palo Alto, CA, USA) was used to identify precise breakpoints of the inserted PLP1-containing X chromosome segment into 1p36. According to the manufacturer's protocol, the genomic DNA of the patient was completely digested with four separate restriction enzymes (i.e. EcoRV, DraI, PvuII, and SspI) and subsequently ligated to the GenomeWalker adaptors. The GenomeWalker libraries produced were then used as a template for primary PCR amplification. The primers used were the outer adaptor primer provided in the kit and a Xq22 specific primer localized at the boundaries of the duplicated PLP1 segment identified with our highly resolutive PLP1-custom array CGH. A secondary nested PCR using the nested adaptor primer and a nested Xq22 specific primer was then performed. Sequencing of the PCR products was finally achieved if a single or two (considering that both, the normal Xq22 and the 1 p36 derivative, alleles could be digested and ligated to the adaptor) specific and major PCR products were visible after electrophoresis through an agarose gel stained with GelRed (Biotium, Hayward, CA, USA). Sequencing was conducted using the nested primers with the BigDye terminator sequencing kit (Applied Biosystems).

\section{Results}

\section{Case report}

Here we report the case of a girl who is the third child from unrelated and healthy parents of Greek origin. Pregnancy was uneventful, however during the second month of life a horizontal nystagmus and severe hypotonia were noticed. At 9 months of age, a brain magnetic resonance imaging (MRI) was performed due to a persistent delay in motor milestones associated with ataxia of the head and trunk led; MRI revealed a severe delay in the myelination process (evaluated at 2 months of age). Afterwards, she improved obviously in motor capacities (holding her head up at 12 months, sitting with support at 16 months, and standing position at 30 months) with excellent psychosocial interactions and decreased nystagmus intensity. Another brain MRI at 33 months of age confirmed the diffuse "hypomyelinating" pattern of the supratentorial cerebral white matter (Fig. 1). In addition, brainstem auditory evoked potentials showed an exclusive cochlear wave I without subsequent recordable brain waves suggesting a severe impairment in CNS conduction. No other abnormalities including dysmorphic features, heart, kidney, or liver problems were found. Now aged 4 years, she continues to improve (crawling, walking with support, feeding herself, drawing a line, speaking using comprehensive simple sentences). However, signs of pyramidal tract dysfunctions are clearly present in the lower limbs and MRI abnormalities have not changed. Those clinical features strongly suggested the diagnosis of PMD.

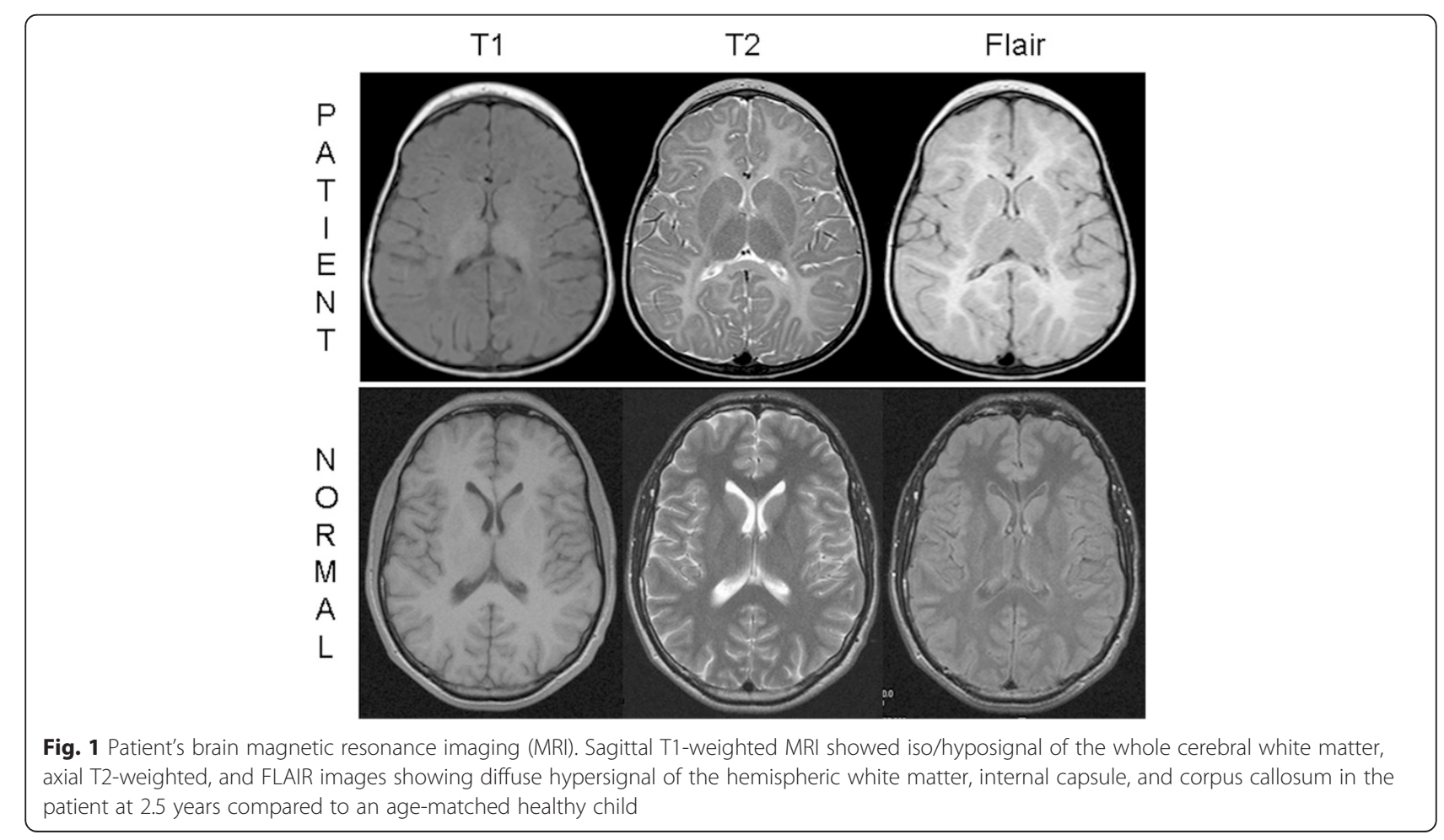




\section{Characterization of the PLP1 gene duplication}

MLPA analysis of the DNA extracted from the patient's blood revealed a duplication of the seven probes localized in each coding exon of the PLP1 gene. Additional probes that were duplicated revealed that the duplication extended at least from the TCEAL1 to ESX1 genes thus encompassing at least $614 \mathrm{~Kb}$ (but less than $1685 \mathrm{~Kb}$ ).

To confirm the PLP1 duplication and define more precisely the size of the duplicated segment we used a custom array $\mathrm{CGH}(\mathrm{aCGH})$ designed to encompass the Xq22 genomic region containing the PLP1 gene with maximum resolution. The aCGH profile confirmed the MLPA result and specified the breakpoint of the duplicated genomic segment covering from $102,761,000$ to $103,513,000$ (752 $\mathrm{Kb}$ of length) on Xq22 according to the GRCh37/hg19 assembly (Fig. 2a).
We next performed FISH analysis with a BAC probe specific to the PLP1 locus (RP11-832L2, Xq22.2) on lymphocyte metaphase spreads. Three distinct PLP1specific signals were visualized consistent with the PLP1 duplication in a girl. Two probes bound to the normal $\mathrm{Xq} 22$ band of both $\mathrm{X}$ chromosomes together with an additional ectopic signal that bound just proximal to the CEB108/T7 1p36 subtelomeric probe (Fig. 2b). Another FISH analysis with BAC probes localized to X chromosome telomeres did not reveal a translocation involving the $\mathrm{X}$ chromosome. Hence, the additional copy of PLP1 results from an insertion into chromosome 1 p36 rather than a classical translocation. A whole genome microarray confirmed the PLP1 duplication but did not reveal any copy number alteration in 1 p36 region. MLPA analysis in the parents did not reveal any variation in PLP1 copy

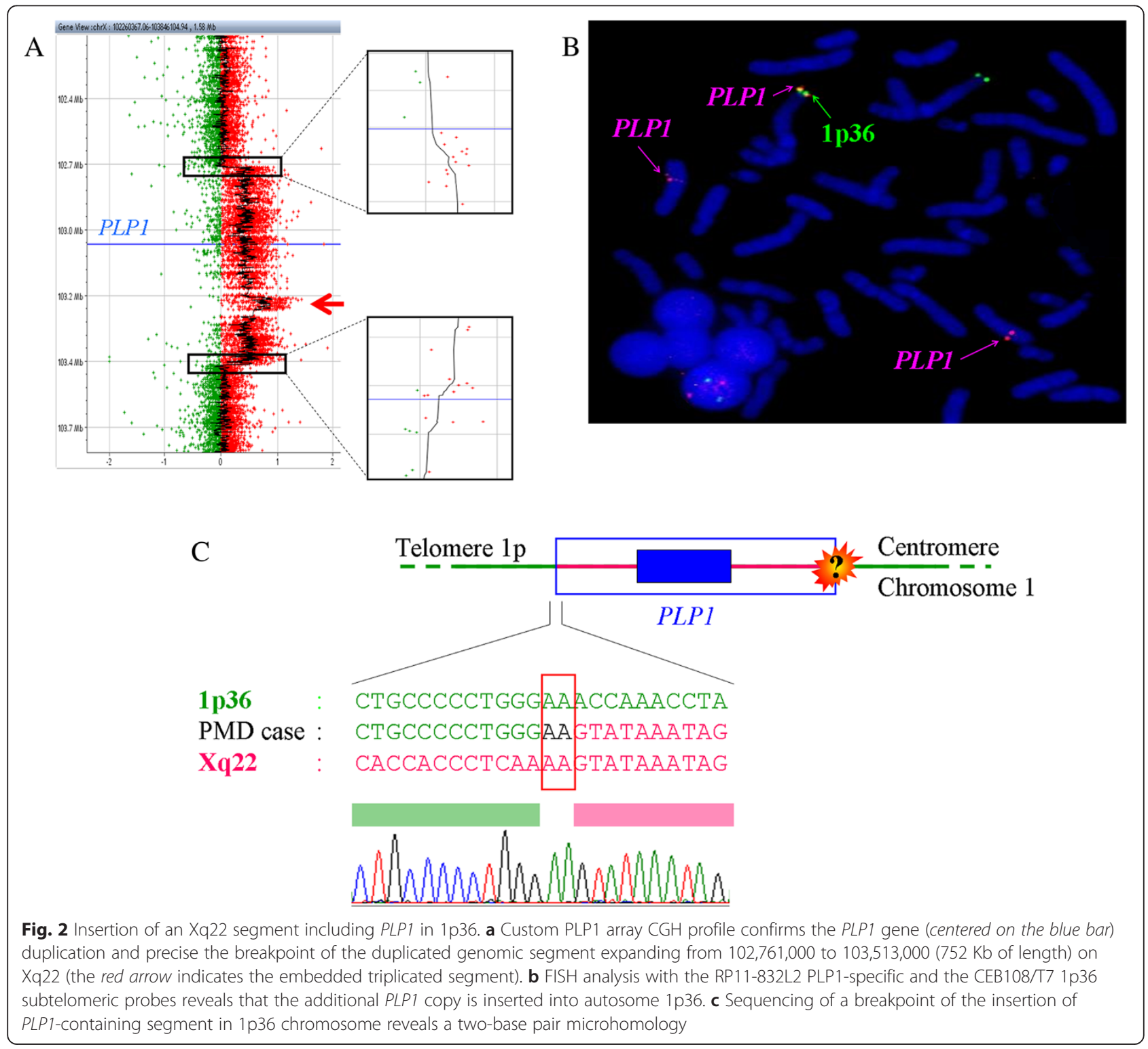


number. However, we wondered if this rearrangement was nevertheless inherited from a parental balanced rearrangement. Therefore we performed the PLP1 specific FISH analysis on the parents' blood and did not detect any ectopic localization of the PLP1 gene. We thus conclude that this rearrangement was acquired either in the gametes of one of the parents or de novo.

\section{Absence of skewed $\mathrm{X}$ inactivation pattern}

A skewed $\mathrm{X}$ inactivation pattern resulting in inactivation of the $\mathrm{X}$ chromosome carrying the abnormal PLP1 gene has been suggested to explain why most females who carry PLP1 genomic alteration are asymptomatic [12]. We thus performed an analysis of the $\mathrm{X}$ inactivation pattern based on the extend HUMARA assay [8]. This analysis of DNA extracted from a blood sample from the patient did not reveal a significant skewed pattern for any of the four $\mathrm{X}$ chromosome loci explored (i.e. $A R$, PCSK1N, SLITRK4, and ZDHHC15). Although we cannot assert that there is not a skewed $\mathrm{X}$ chromosome inactivation pattern in the oligodendrocytes of the patient, the insertion of an extra-copy of PLP1 in an autosome did lead to a functional duplication irrespective of the Xinactivation pattern.

\section{PLP1 over-expression in patient fibroblasts}

We also quantified the PLP1 gene expression in fibroblasts from the patient and compared it to expression in normal control fibroblasts and fibroblasts from male patients with classical tandem PLP1 duplications. The duplicated controls and our patient showed a 1.88 and a 5.21-fold increase of the PLP1 expression respectively compared to the normal control. This result confirmed the functional duplication of the PLP1 gene leading to its overexpression in the patient's fibroblasts.

\section{Precise mapping of the breakpoint using genome walking strategy}

To further characterize the insertion site of PLP1 on chromosome $1 \mathrm{p} 36$, we performed a genome walking strategy. This molecular procedure allows sequencing of the 1 p36 telomeric flanking segment directly adjacent to the breakpoint of the duplicated genomic region of Xq22 (Fig. 2c). The Xq22 segment inserted exactly at the position chr1:140,332 (GRCh37/hg19) on chromosome 1p36. In addition, we identified 2 bp of microhomology shared by the $1 \mathrm{p} 36$ and the Xq22 boundaries genomic sequences.

\section{Discussion}

We have described the case of a girl with a classical form of PMD due to an insertion of the PLP1 gene into $1 \mathrm{p} 36$ autosome. PLP1 gene duplication in boys results in PLP overexpression and subsequent accumulation in the cytoplasm of the myelinating oligodendrocytes, leading to a CNS myelin defect. We demonstrated that the PLP1 transcript is overexpressed in the fibroblasts of our patient with an insertion of an additional copy of PLP1 in chromosome $1 \mathrm{p} 36$. It has been proposed that PLP1 expression in fibroblasts is a good cellular model for reflecting the consequences of an altered PLP1 gene dosage [9]. We thus assume that the additional copy of PLP1 led to an overexpression of PLP in oligodendrocytes and that subsequent toxicity in this cell type was responsible for the neurological phenotype. The PLP1 overexpression seems even higher in our patient's fibroblasts compared to classical tandem duplications in boys. This could be related to the epigenetic state of the chromatin environment in the $1 \mathrm{p} 36$ insertion site that could be more favorable to gene expression than the Xq22 PLP1 locus.

Sequence analysis of the boundaries of the Xq22 duplicated segment revealed two nucleotides of microhomology at the telomeric breakpoint junction (Fig. 2c) consistent with a replication based mechanism, such as fork stalling and template switching (FoSTeS) [10] or microhomologymediated break-induced replication (MMBIR) [13]. We cannot rule out that the two-base microhomology observed at the breakpoint occurs by chance and is not related to a replicative-based mechanism. However, it is noteworthy that James Lupski and colleagues firstly described the elegant FoSTeS mechanism precisely at the PLP1 locus [10]. Based on this model, we propose a genomic mechanism to explain the microhomology-based insertion of PLP1 in an autosome (Fig. 3): instead of switching to a template on the sister chromatid, as it has been classically described, the replication fork may switch to chromosome 1p36. Indeed, in the classical model (i.e. intrachomosomal rearrangement), both replication forks may be separated by large genomic sequence leading to extensive duplication or deletion. It is reasonable to believe that the new replication fork reestablish by considering the physical proximity of the new microhomology-based site. Thus, the new replication fork could take place on distinct chromosome if the insertion site is physically close considering the threedimensional structure of the chromatin in interphase nuclei. This may be supported by the fact that another PLP1 rearrangement involving the $1 \mathrm{p} 36$ genomic region has already been described [5].

Despite several primer combinations and PCR conditions we were unable to obtain the second breakpoint junction. The inability to obtain a PCR product for the centromeric breakpoint may be related to the complexity of the rearrangement at this breakpoint. Indeed, the PLP1-custom aCGH revealed a triplicated segment embedded in the PLP1 duplication from chrX:103,230,500 to chrX:103,317,500 (Fig. 2a). Triplications of the PLP1 


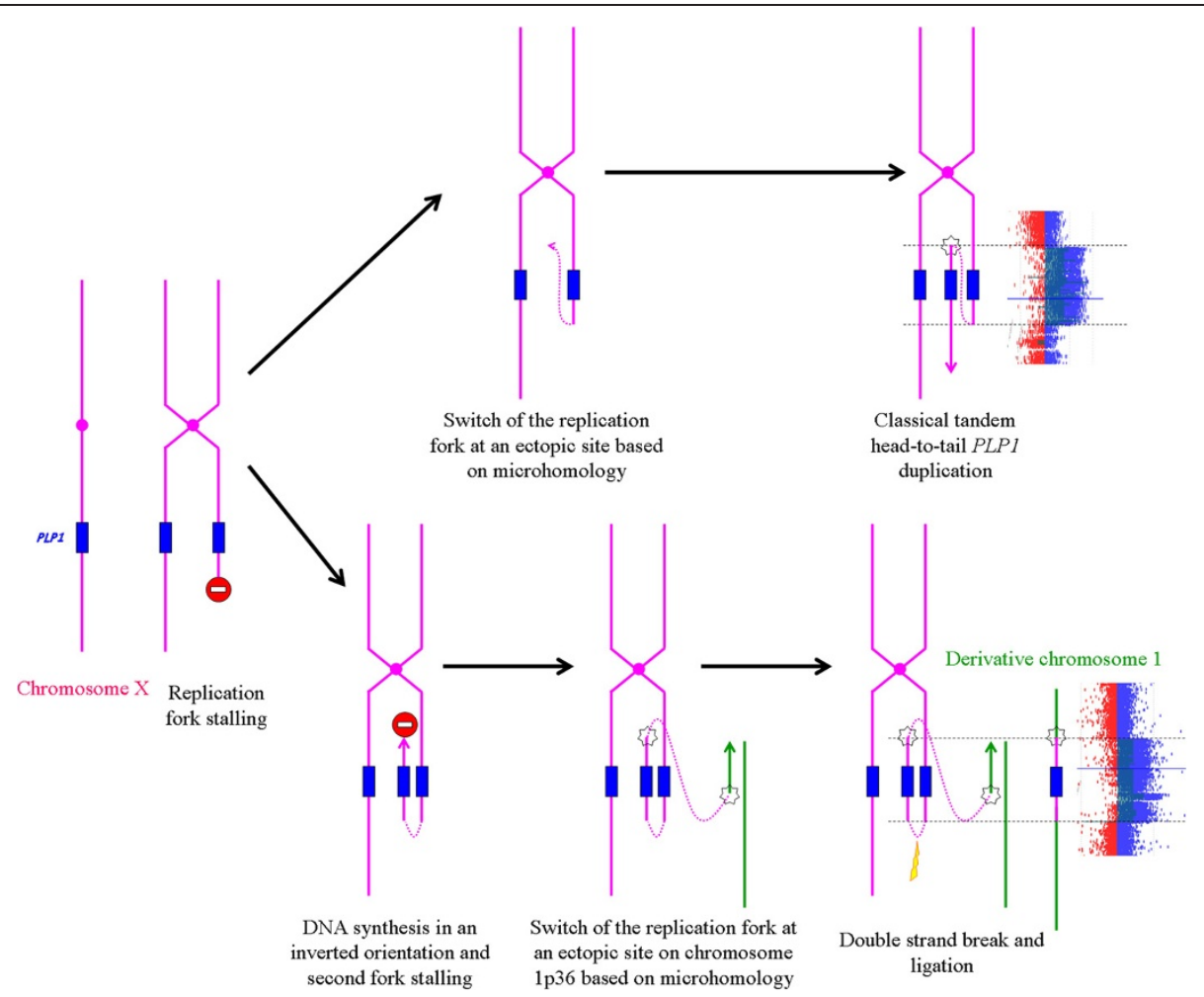

Fig. 3 Hypothetical model of mechanism for the insertion of PLP1 in 1p36. Based on the FoSTeS mechanism proposed by Lupski and coll. [10] (upper situation) we propose an alternative model to explain the insertion of an extra copy of the PLP1 gene in chromosome 1 p36 (lower situation)

gene have already been described and are associated with a more severe phenotype compared with patients with duplications [2,3]. However, in our case, the triplicated segment did not include the PLP1 gene. This is consistent with the mild phenotype of our patient compared to PMD boys with three copies of PLP1. It has been proposed that triplications at the PLP1 locus result from a duplication-inverted triplication-duplication (DUP-TRP/ INV-DUP) likely based on a replication based mechanism and that relies on the presence of segmental duplications in an inverted orientation [14]. Interestingly, the triplicated segment observed in our case occurred between two segmental duplications encompassing the H2BFXP pseudogenes localized $60 \mathrm{~Kb}$ from each other in an inverted direction. This triplicated segment further illustrates the complexity of the observed rearrangement.

\section{Conclusion}

Here we describe the case of a girl with a classical form of PMD due to an insertion of a $752 \mathrm{~Kb}$ Xq22 region containing the whole PLP1 gene into 1p36 autosome. This rearrangement harbors a two base-pair microhomology at one of the breakpoint suggesting a replication based mechanism such as FoSTeS or MMBIR. In addition to PLP1 duplications, other genomic rearrangements responsible for PMD, including deletions [15, 16], and translocations [4] have been described. Complex genomic rearrangements including duplication-inverted triplications $[2,3]$ and complex duplications consisting of two duplicated fragments interspersed with a segment without copy number variation [6] have also been identified at the PLP1 locus. Our result further emphasizes the large diversity of the genomic rearrangement involving the PLP1 gene probably based on the complexity of the genomic architecture [7]. Finally, from a clinical point of view, careful consideration should be given to girls with classical PMD clinical features since they usually experience complex PLP1 genomic alteration with distinct risk of inheritance. FISH analysis should thus be considered, in addition to copy number evaluation and PLP1 gene sequencing, to detect those complex rearrangements in girls with PMD.

\section{Competing interests}

All authors certify that there is no conflict of interest regarding the material discussed.

\section{Authors' contributions}

JMP , CP, ID, SD and OBT participated in the design of the study, and interpretation of data. CD and ACT performed FISH analysis. EEP and MGB performed the GRT-PCR analysis. AT and SQ performed the CGH analysis. GV, FR, CK, EK, and OBT provided clinical follow-up. ME, GV, FR, and OBT read the 
brain MRI. JMP wrote the manuscript with assistance from OBT. All authors read and approved the final manuscript.

\section{Acknowledgements}

This work was supported by grants from ELA Foundation (2009-00714AV2) and "Les amis de lanis" association (Ploudaniel, France).

Written informed consent was obtained from the patient's legal guardian(s) for gene analysis and for collection of data.

\section{Author details}

${ }^{1}$ UF de Génétique moléculaire, Hôpital Robert Debré, AP-HP, Paris, France. Inserm U1141, Université Paris Diderot, Sorbonne Paris Cité, Hôpital Robert Debré, Paris, France. ${ }^{3} \mathrm{New}$ adresse: Unité de Génétique Somatique, Institut Curie, Paris, France. ${ }^{4}$ UF de Cytogénétique, Hôpital Robert Debré, AP-HP, Paris, France. ${ }^{5}$ Paediatric Neurology, P\&A Kyriakou Paed. Hospital, Athens Medical School, Athens, Greece. 'Cytogénétique Médicale, Univ Clermont1, UFR Médecine, CHU Estaing, Clermont-Ferrand, France. ' ERTICa, EA 4677, Univ Clermont1, UFR Médecine, Clermont-Ferrand, France. ${ }^{8}$ Neurologie et maladie métabolique, Hôpital Robert Debré, AP-HP, Paris, France. ${ }^{9} \mathrm{IUH}$, Hôpital Saint-Louis, Paris, France. ${ }^{10}$ Imagerie pédiatrique, Hôpital Robert Debré, AP-HP, Paris, France.

Received: 10 February 2015 Accepted: 25 August 2015

Published online: 02 September 2015

\section{References}

1. Woodward KJ. The molecular and cellular defects underlying PelizaeusMerzbacher disease. Expert Rev Mol Med. 2008;10:e14.

2. Wolf NI, Sistermans EA, Cundall M, Hobson GM, Davis-Williams AP, Palmer R, et al. Three or more copies of the proteolipid protein gene PLP1 cause severe Pelizaeus-Merzbacher disease. Brain J Neurol. 2005;128(4):743-51.

3. Shimojima K, Mano T, Kashiwagi M, Tanabe T, Sugawara M, Okamoto N, et al. Pelizaeus-Merzbacher disease caused by a duplication-inverted triplication-duplication in chromosomal segments including the PLP1 region. Eur J Med Genet. 2012;55(6-7):400-3.

4. Fonseca ACS, Bonaldi A, Costa SS, Freitas MR, Kok F, Vianna-Morgante AM. PLP1 duplication at the breakpoint regions of an apparently balanced $t(X ; 22)$ translocation causes Pelizaeus-Merzbacher disease in a girl. Clin Genet. 2013;83(2):169-74

5. Yiu EM, Farrell SA, Soman T. Classic Pelizaeus-Merzbacher disease in a gir with an unbalanced chromosomal translocation and functional duplication of PLP1. Mov Disord. 2009;24(14):2171-2.

6. Carvalho CM, Bartnik M, Pehlivan D, Fang P, Shen J, Lupski JR. Evidence for disease penetrance relating to CNV size: Pelizaeus-Merzbacher disease and manifesting carriers with a familial $11 \mathrm{Mb}$ duplication at Xq22. Clin Genet. 2012;81(6):532-41.

7. Dittwald P, Gambin T, Gonzaga-Jauregui C, Carvalho CM, Lupski JR, Stankiewicz P, et al. Inverted low-copy repeats and genome instability-a genome-wide analysis. Hum Mutat. 2013;34(1):210-20.

8. Bertelsen $B$, Tümer $Z$, Ravn K. Three new loci for determining $X$ chromosome inactivation patterns. J Mol Diagn. 2011;13(5):537-40.

9. Regis S, Grossi S, Corsolini F, Biancheri R, Filocamo M. PLP1 gene duplication causes overexpression and alteration of the PLP/DM20 splicing balance in fibroblasts from Pelizaeus-Merzbacher disease patients. Biochim Biophys Acta. 2009;1792(6):548-54.

10. Lee JA, Carvalho CMB, Lupski JR. A DNA replication mechanism for generating nonrecurrent rearrangements associated with genomic disorders. Cell. 2007;131(7):1235-47.

11. Regis S, Biancheri R, Bertini E, Burlina A, Lualdi S, Bianco MG, et al. Genotype-phenotype correlation in five Pelizaeus-Merzbacher disease patients with PLP1 gene duplications. Clin Genet. 2008;73(7):279-87.

12. Woodward K, Kirtland K, Dlouhy S, Raskind W, Bird T, Malcolm S, et al. $X$ inactivation phenotype in carriers of Pelizaeus-Merzbacher disease: skewed in carriers of a duplication and random in carriers of point mutations. Eur J Hum Genet. 2000;8(6):449-54.

13. Hastings PJ, Ira G, Lupski JR. A microhomology-mediated break-induced replication model for the origin of human copy number variation. PLoS Genet. 2009;5(1):e1000327.

14. Carvalho CM, Ramocki MB, Pehlivan D, Franco LM, Gonzaga-Jauregui C, Fang $P$, et al. Inverted genomic segments and complex triplication rearrangements are mediated by inverted repeats in the human genome. Nat Genet. 2011:43(11):1074-14081.

15. Inoue K, Osaka H, Thurston VC, Clarke JT, Yoneyama A, Rosenbarker L, et al. Genomic rearrangements resulting in PLP1 deletion occur by nonhomologous end joining and cause different dysmyelinating phenotypes in males and females. Am J Hum Genet. 2002;71(4):838-53.

16. Torisu H, Iwaki A, Takeshita K, Hiwatashi A, Sanefuji M, Fukumaki Y, et al. Clinical and genetic characterization of a 2-year-old boy with complete PLP1 deletion. Brain Dev. 2012;34(10):852-6.

\section{Submit your next manuscript to BioMed Central and take full advantage of:}

- Convenient online submission

- Thorough peer review

- No space constraints or color figure charges

- Immediate publication on acceptance

- Inclusion in PubMed, CAS, Scopus and Google Scholar

- Research which is freely available for redistribution 